\title{
Atrophic upper pole of a duplex collecting system masquerading as suprarenal mass: a case study and literature review
}

\author{
Alex Kavanagh, MD; lain McAuley, MD, FRCSC; Michelle Longpre, BSc Hon, MD; Andrew E. MacNeily, MD, \\ FRCSC, FAAP
}

\section{Abstract}

The growing use of maternal fetal ultrasound is leading to the discovery of an increasing number of suprarenal masses. Our experience with a cystic suprarenal mass detected on antenatal ultrasound is described. Location and radiographic features could not rule out the possibility of a cystic neuroblastoma; therefore, surgical resection of the mass was performed. Despite the absence of common radiologic characteristics, the pathology of the specimen revealed a non-functioning upper pole of a duplex kidney with complete duplication of the collecting system. Neonatal evaluation and management and the differential diagnosis are discussed.

Can Urol Assoc J 2010;4(4):E94-96

\section{Case report}

A routine second trimester ultrasound examination of a 28-year-old female (gravida 2, para 1) showed a well circumscribed, hypoechoic mass on the upper pole of the right fetal kidney. The mass measured about $6 \mathrm{~cm}$ in its largest diameter and appeared predominantly cystic in structure. The remainder of the detailed sonographic evaluation was unremarkable and no ureterectasis or ureterocele was observed. Size, echogenecity and structure of the mass remained unchanged throughout the pregnancy on repeated scans. An uneventful vaginal delivery at 38 weeks gestational age resulted in a female child with healthy Apgar scores.

Postpartum examination of the neonate was completely normal, without palpable abdominal mass or lumbosacral abnormality. Abdominal ultrasound at 3 days demonstrated that the cystic structure remained unchanged in size and appeared contiguous with the upper pole of the kidney. The perinatal period was complicated with a simple, culture-positive urinary tract infection at 4 months, which was treated with oral cephalexin. The child was followed with serial ultrasounds at 3 month intervals during her first year of life (Fig. 1). Throughout this period, the cyst was noted to increase in size by a small amount (less than $1 \mathrm{~cm}$ in greatest diameter), but remained unchanged in echogenicity and structure. Renal size was comparable bilaterally throughout this period.

At 11 months, the child was admitted to hospital with a second culture-positive urinary tract infection and treated with intravenous ampicillin and gentamicin. An ultrasound performed during her admission suggested that the cystic structure was more likely suprarenal in origin and displacing the right kidney. Subsequent imaging with a voiding cystogram was normal. A magnetic resonance imaging (MRI) scan at this time further suggested suprarenal origin of the cyst with compression and anterior displacement of the upper pole of the right kidney (Fig. 2). Neither hydroureter nor ureterocele was observed on MRI.

Based on the location and radiologic appearance of the cyst, our differential diagnosis included simple renal cyst, duplex kidney and adrenal neuroblastoma. Biochemical evaluation revealed elevated urinary catecholamines (urine dopamie 735 umol/molCr [normal 175-735]; urine epinephrine $23.3 \mathrm{umol} / \mathrm{molCr}$ [normal 2.5-1.3]; urine norepinephrine $72 \mathrm{umol} / \mathrm{molCr}$ [normal 14-56]) and normal urine metanephrines (urine metanephrine $0.114 \mathrm{mmol} / \mathrm{molCr}$ [normal 0.023-0.302]; urine normetanephrine $0.353 \mathrm{mmol} / \mathrm{mol} \mathrm{Cr}$ [normal 0.216-0.787]). Due to the inability to eliminate neuroblastoma from the differential diagnosis, the patient was referred to our centre for surgical resection of the mass at 15 months. Via a flank approach, we explored the retroperitoneum. A complete duplication of the right collecting system was encountered, with a cystic upper pole moiety. An uncomplicated right upper pole heminephrectomy was performed. Repeat urinary catecholamines at the time of surgery revealed normal levels. The child recovered well from the procedure and was discharged in stable condition shortly afterwards. Pathologic evaluation of the specimen revealed a dysplastic and atrophic upper pole of a duplex kidney with complete duplication of the collecting system. 


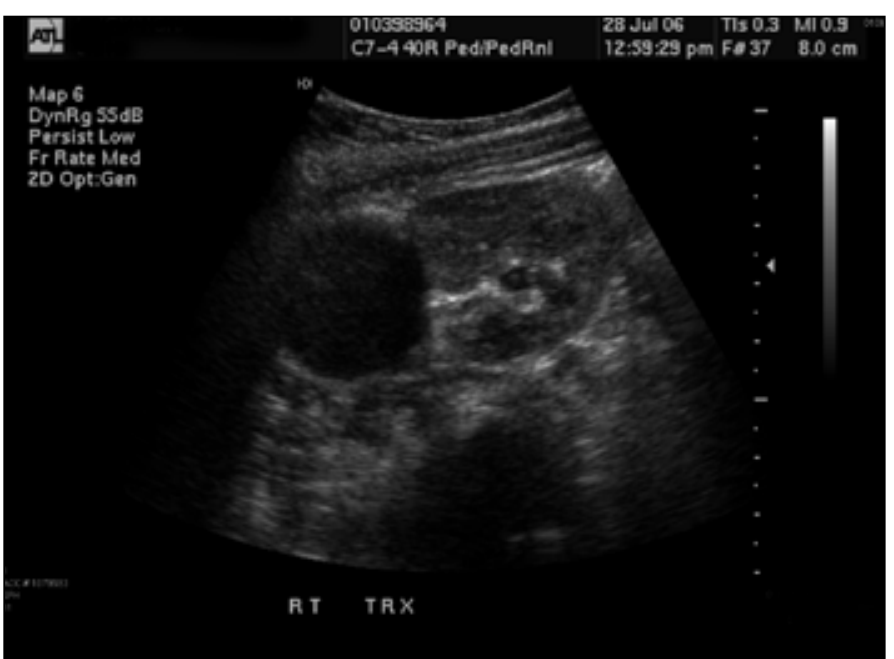

Fig. 1. An ultrasound evaluation of the cystic structure superior to the right kidney at 10 months of age.

\section{Discussion}

Radiologic evaluation of our patient demonstrated a 6-cm unilocular, suprarenal mass without solid components or internal septation that did not change appreciably in size during the first year of life. Neither ultrasound nor MRI studies were able to demonstrate hydroureter or ureterocele. Our differential diagnosis included duplex collecting system with upper pole dysplasia, simple renal cyst and suprarenal neuroblastoma. Other possible diagnoses for antenatal suprarenal cysts include benign entities, such as adrenal hemorrhage, retropleural pulmonary sequestration, neurogenic cysts, enteric duplication cysts and malignant diseases, including Wilm's tumour and congenital mesoblastic nephroma. ${ }^{1,2}$ Lack of evolution of the lesion in addition to consistent hypoechoic density without solid components reduced the likelihood of these diagnoses.

The antenatal sonographic characteristics most accurately associated with a duplex kidney include cysts in the upper pole of the kidney; the presence of 2 separate non-communicating renal pelves; a dilated ureter (usually draining the upper pole); and echogenic cystic structures in the bladder (ureterocele). ${ }^{3}$ Detection of 2 separate poles or ureterocele are the strongest predictors of duplex renal units with predictive power of $93 \%$ and $78 \%$, respectively. ${ }^{4}$ Antenatal ultrasound with a duplex kidney may only show cystic dysplasia, leading to unclear differentiation between masses of renal and suprarenal origin.

Postnatal symptoms of duplex collecting systems during the first year of life include urinary tract infections and chronic pyelonephritis. If the duplex system is associated with ectopic ureter then infection, urinary retention, dribbling incontinence and abdominal mass or prolapsing ureterocele may lead to the diagnosis. ${ }^{5}$ Voiding cystourethrography (VCUG) demonstrates reflux in about $66 \%$ of cases of

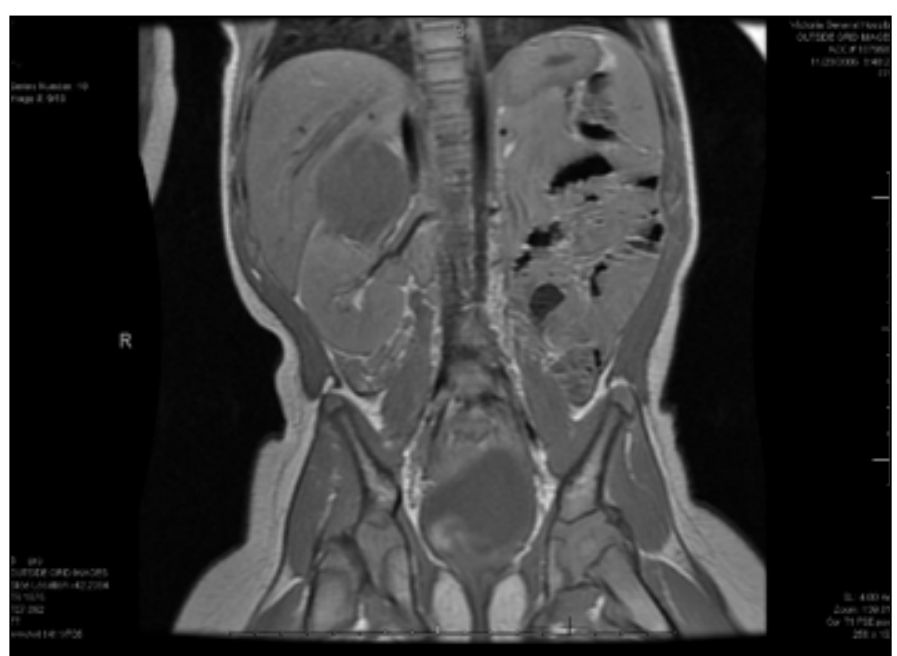

Fig. 2. T1-weighted magnetic resonance imaging assessment of the cystic structure superior to the right kidney at 12 months of age.

complete duplication and ureterocele in $6 \%$ of completely duplicated systems. ${ }^{6}$ Renal scarring may be assessed with either dimercaptosuccinic acid (DMSA) or mercaptoacetyltriglycine (MAG3) renal scans. The treatment of duplex collecting systems is based on the degree of renal impairment and morbidity. For simple duplex systems with no dilatation of either moiety and with no evidence of obstruction or reflux, no treatment is needed. Heminephrectomy should be considered for complex duplex systems where there is increasing renal damage, failure of antibiotic prophylaxis or a poorly functioning moiety (less than $10 \%$ ). ${ }^{5}$

Neuroblastoma is a tumour of the postganglionic sympathetic neurons and is the most common extracranial solid tumour of childhood with a prevalence of about 1 case per 7000 births. $^{7}$ More than $90 \%$ of prenatally diagnosed neuroblastomas are adrenal in origin. In contrast, only $35 \%$ of all neuroblastomas diagnosed in infants and young children are adrenal in origin. ${ }^{8}$ Adrenal neuroblastoma is commonly manifested as a suprarenal mass having a solid, cystic or mixed sonographic appearance which may contain areas of calcification. ${ }^{9}$ Homovanillic acid (HVA), vanillylmandelic acid (VMA) and acidic metabolites of catecholamines are excreted in higher than normal amounts by patients with neuroblastoma, and can therefore be used as tumour markers for diagnosis. ${ }^{10}$ Unfortunately, HVA and VMA levels are less sensitive in antenatally diagnosed neuroblastoma, compared with postnatally diagnosed patients. ${ }^{9,10}$ Methyliodobenzylguanidine (MIBG) scintigraphy can be used to identify neuroblastoma, but its utility is limited by the fact that only $70 \%$ of perinatal neuroblastomas are MIBG avid and the negative predictive power is $55 \% .{ }^{11}$

The management of perinatal neuroblastoma is based on the International Neuroblastoma Staging System (INSS) criterion in addition to radiologic examination with computed tomography and MIBG scintigraphy. In most cases, infants 
with localized primary tumours (Stage 1 disease) undergo complete gross excision and have overall survival rates in excess of $95 \% .^{7}$ The excellent outcome of infants with localized, resectable disease coupled with the observation that some tumours seen on antenatal imaging spontaneously regress has prompted investigators to consider managing a subset of patients with expectant observation rather than surgical resection. ${ }^{11}$ Small and purely cystic neuroblastomas are believed to have favourable prognosis due to similar histological characteristics with in situ neuroblastomas which have a high rate of spontaneous regression. ${ }^{11}$ The Children's Oncology Group has initiated a prospective, single arm clinical trial of expectant observation for infants with small stage 1 neuroblastomas diagnosed either prenatally or in the first 6 months of life to determine the feasibility of expectant management of tumours with favourable biologic characteristics. The main disadvantage of an observation strategy is that it increases the risk that a stage 1 patient will require salvage chemotherapy for disease progression. ${ }^{11}$

\section{Conclusion}

The infant with a prenatal diagnosis of a cystic suprarenal mass that remains constant in size, with a elevated urinary catecholamines result, can present a significant diagnostic dilemma. Our case demonstrates that duplex kidney must be considered on the differential diagnosis of a suprarenal mass even in the absence of characteristic radiologic signs. Both sonographic and MRI assessment of our patient failed to demonstrate presence of 2 non-communicating renal pelves, dilated ureter or ureterocele which would be predictive of a duplex system. Further investigation with VCUG did not demonstrate reflux and confirmed the absence of ureterocele. Ultrasound morphology was suggestive of a suprarenal mass consistent with a purely cystic neuroblastoma, a simple renal cyst or a duplex system with upper pole dysplasia. Only surgical resection and pathologic examination were able to confirm the presence of a dysplastic upper pole of a duplex kidney with duplication of the collecting system.

Department of Urologic Sciences, UBC, Vancouver, BC

Competing interests: None declared.

This paper has been peer-reviewed.

\section{References}

1. Brame M, Masel J, Homsy Y. Antenatal detection and management of suprarenal masses. Urology 1999;54:1097.

2. Rubenstein $S C$, Benacerraf $B R$, Retik $A B$, et al. Fetal suprarenal masses: sonographic appearance and differential diagnosis. Ultrasound Obstet Gynecol 1995;5:164-7.

3. Abuhamad AZ, Horton CE Jr, Horton SH, et al. Renal duplication anomalies in the fetus: clues for prenatal diagnosis. Ultrasound Obstet Gynecol 1996;7:174-7.

4. Whitten SM, McHoney M, Wilcox DT, et al. Accuracy of antenatal fetal ultrasound in the diagnosis of duplex kidneys. Ultrasound Obstet Gynecol 2003;21:342-6.

5. Whitten SM, Wilcox DT. Duplex systems. Prenat Diagn 2001;21:952-7.

6. Siomou E, Papadopoulou F, Kollios KD, et al. Duplex collecting system diagnosed during the first 6 years of life after a first urinary tract infection: a study of 63 children. J Urol 2006;175:678-81; discussion 681-2.

7. Weinstein JL, Katzenstein HM, Cohn SL. Advances in the diagnosis and treatment of neuroblastoma. Oncologist 2003;8:278-92.

8. Acharya S, Jayabose S, Kogan SJ, et al. Prenatally diagnosed neuroblastoma. Cancer 1997;80:304-10.

9. Ho PTC, Estroff JA, Kozakewich H, et al. Prenatal detection of neuroblastoma: a ten-year experience from the Dana-Farber Cancer Institute and Children's Hospital. Pediatrics 1993;92:358-64.

10. Tuchman M, Ramnaraine MLR, Woods WG, et al. Three years of experience with random urinary homovanillic and vanillymandelic acid levels in the diagnosis of neuroblastoma. Pediatrics 1987;79:203-5.

11. Nuchtern JG. Perinatal neuroblastoma. Semin Pediatr Surg 2006;15:10-6.

Correspondence: Dr. Alex Kavanagh, Department of Urologic Sciences, Faculty of Medicine, UBC, Gordon \& Lesie Diamond Health Care Centre Level 6, 2775 Laurel St., Vancouver, BC V5Z 1M9; fax: 604-875-4637; alexkava@interchange.ubc.ca 